\title{
Analysis of Multiple Coulomb Scattering of Muons in the MICE Liquid $\mathrm{H}_{2}$ Absorber
}

\author{
Gavriil Chatzitheodoridis $^{a, b, c, *}$ on behalf of the MICE collaboration ${ }^{* *}$ \\ ${ }^{a}$ SUPA and the Department of Physics, University of Strathclyde, 107 Rottenrow, Glasgow, UK \\ ${ }^{b}$ School of Physics and Astronomy, Kelvin Building, University of Glasgow, Glasgow, UK \\ ${ }^{c}$ Cockcroft Institute, Daresbury Laboratory, Sci-Tech Daresbury, Keckwick Ln, Daresbury, Warrington, \\ $U K$ \\ E-mail: gavriil.t.chatzitheodoridis@strath.ac.uk
}

The Muon Ionization Cooling Experiment (MICE), is a multi-national accelerator physics experiment created to demonstrate Ionization Cooling (IC); a new, rapid beam-cooling technique suitable for the short-lived muon. The performance of IC depends on two key processes - energy loss due to collisional ionization, and Multiple Coulomb Scattering (MCS) - for which accurate models are crucial in enabling quantitative design studies for future muon accelerators. Experimental measurements of MCS of positive muons with momenta in the range 170-240 MeV/c in liquid hydrogen are reported in this paper.

\footnotetext{
*** The European Physical Society Conference on High Energy Physics (EPS-HEP2021), ***

*** 26-30 July $2021 * * *$

*** Online conference, jointly organized by Universität Hamburg and the research center DESY ***
}

\footnotetext{
** The work described here was made possible by grants from the Department of Energy and National Science Foundation (USA), the Istituto Nazionale di Fisica Nucleare (Italy), the Science and Technology Facilities Council (UK), the European Community under the European Commission Framework Programme 7 (AIDA project, grant agreement no. 262025, TIARA project, grant agreement no. 261905, and EuCARD), the Japan Society for the Promotion of Science and the Swiss National Science Foundation, in the framework of the SCOPES programme. We gratefully acknowledge all sources of support. We are grateful to the support given to us by the staff of the STFC Rutherford Appleton and Daresbury Laboratories and the Cockroft Institute. We acknowledge the use of Grid computing resources deployed and operated by GridPP in the UK, http://www.gridpp.ac.uk/.

*Speaker
} 


\section{Introduction}

High-brightness muon beams may be used in future accelerator physics facilities for a range of research topics [1,2]. Although the muon is an attractive candidate due to reduced Bremsstrahlung losses, the primary challenge is its short life-time $(2.2 \mu$ s at rest) and the relatively long cooling periods required by the current techniques to obtain high-brightness beams.

\subsection{Ionization Cooling}

A rapid beam cooling technique that exploits momentum loss due to ionizing collisions when a focused beam passes through a low atomic number $(Z)$, high energy absorbent material was recently demonstrated in a particle-by-particle measurement of emittance [3] by the MICE collaboration. To evaluate the performance of future proposed facilities utilising IC, accurate modelling of both collisional ionization and MCS are needed. Past comparisons of predictive models of MCS and experimental measurements have been shown to differ [4]. MCS is the phenomenon describing the multiple small-angle scatters a charged particle undergoes when traversing a material. Equation (1) describes the RMS scattering width (of the central $98 \%$ of the distribution) for the plane projected angle $\theta_{x, y}(y-z, x-z$ plane projection respectively) for a beam with momentum $p$, velocity $\beta=v / c$ traversing some distance $z$ within a material with atomic number $Z$ and radiation length $X_{0}$ [5].

$$
\theta_{R M S}=\frac{13.6 M e V / c}{\beta p} Z \sqrt{\frac{z}{X_{0}}}\left(1+0.038 \frac{z Z^{2}}{X_{0} \beta^{2}}\right)
$$

\section{Method}

\subsection{The MICE Apparatus}

The muons reaching MICE, are decay products of pions provided by the ISIS proton beam colliding with a titanium target. The captured products are momentum selected by a series of magnets before reaching the MICE channel. The MICE Step IV configuration was used for this analysis comprising of the liquid hydrogen container (absorber) - holding 211 of the material at a temperature of $20 \mathrm{~K}$ between the upstream (US) and downstream (DS) scintillating fibre (Sci-Fi) trackers. The cooling channel magnets were not used for the experimental measurements considered in this study (i.e. straight particle tracks in the vicinity of the absorber). Three time-offlight detectors were also used velocity measurements (TOF0, $1 \& 2$ ).

\subsection{Event Selection}

MICE has gathered data with beamline settings configured to provide $170,200 \& 240 \mathrm{MeV} / \mathrm{c}$ muon momenta at the absorber with the vessel empty (but in place) and filled with liquid hydrogen. The data-sets are initially reduced through a set of selection criteria. Only particles that have single space-points (SPs) at each TOF, and a single reconstructed track at the US tracker (bins 2 and 3 of Fig. 1) are considered. Trajectories that are expected to have passed through excessive material (bin 4) or have low reconstruction quality (bin 5) are rejected. Finally, events that are directed at the edges of the DS tracker and those that are outside the selected TOF range are rejected (bin 6 \& 7). 


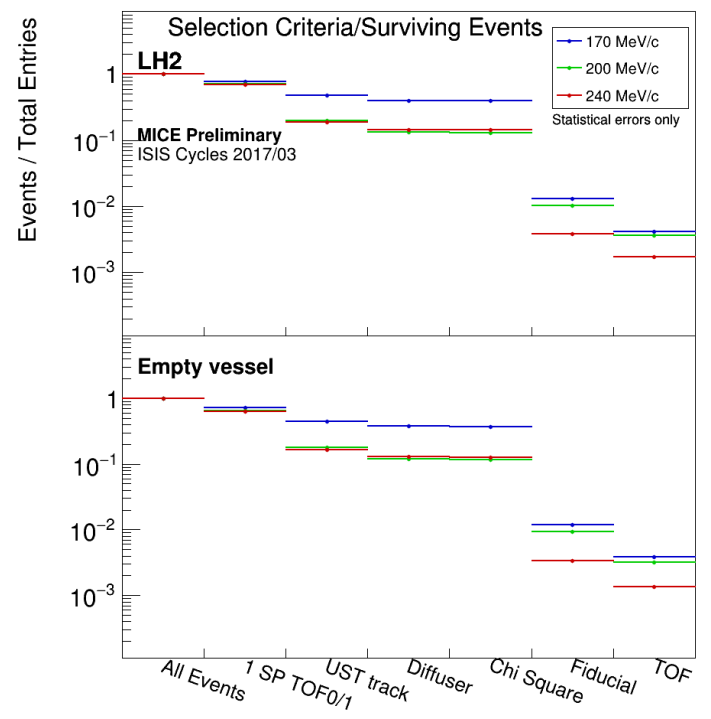

Figure 1: Ratio of events to total number of events (first bin) surviving each cut.

\section{Results}

The resulting scattering distributions of the surviving events (Fig 2) are expressed as the difference of angles between the US and DS momentum vectors $\left(\vec{P}_{U S}, \vec{P}_{D S}\right)$ when projected into corresponding orthogonal planes. The first plane is defined as the plane containing the US vector and the Y experimental axis, and the second plane is defined as its orthogonal that contains $\vec{P}_{U S}$. This means that the two planes required for the projected angle calculations are defined on a particle by particle basis. This definition would be equal to using the $\mathrm{Y}-\mathrm{Z}$ and $\mathrm{X}-\mathrm{Z}$ planes (from experimental coordinates) if all particles moved parallel to the experimental Z-axis. Because this is not the case the following definitions are established for scattering studies:

$$
\begin{gathered}
\theta_{y}=\arctan \left(\frac{\vec{P}_{D S} \cdot\left(\hat{Y} \times \vec{P}_{U S}\right)}{\left|\hat{Y} \times \vec{P}_{U S}\right|\left|\vec{P}_{D S}\right|}\right) \\
\theta_{x}=\arctan \left(\frac{\vec{P}_{D S} \cdot\left(\vec{P}_{U S} \times\left(\hat{Y} \times \vec{P}_{U S}\right)\right.}{\left|\vec{P}_{U S} \times\left(\hat{Y} \times \vec{P}_{U S}\right)\right|\left|\vec{P}_{D S}\right|}\right)
\end{gathered}
$$

\section{Conclusions \& Future work}

This paper presents experimental measurements of MCS of muons crossing a mean of $33 \mathrm{~cm}$ of liquid hydrogen with approximate beam momenta 170, 200, $240 \mathrm{MeV} / \mathrm{c}$ in the MICE channel. The same measurement with identical selection criteria is performed with the liquid hydrogen vessel empty. The scattering distributions for the empty absorber configuration (Fig. 2 bottom) shows the scattering of particles from the intermediate material of the trackers and vessel, mainly scintillator material (BCF-10 plastic fibres), helium and aluminium. The top row shows the added effect of liquid hydrogen. The decrease in width can be observed as the beam momenta increases, whilst the 

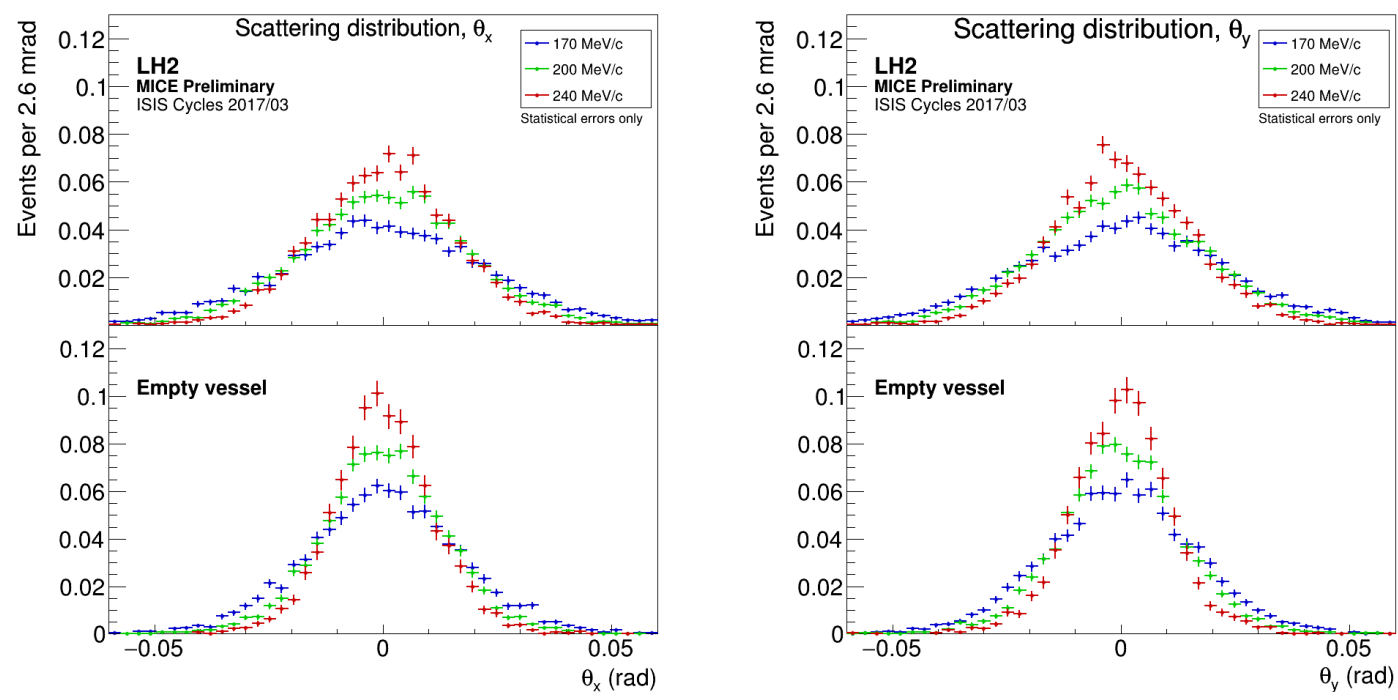

Figure 2: Distribution of $\theta_{x}$ (left) and $\theta_{y}$ (right) for the three momenta settings. For vessel full (top) and empty (bottom) configurations.

width increases at all settings when liquid hydrogen is added in the beam path. The future aim of this analysis is to compare the experimental measurements with the predictions of equation 1 , the GEANT4 [6] simulation and the Moliere [7] MCS model.

\section{References}

[1] D. V. Neuffer, Y. I. Alexahin, M. A. Palmer, C. M. Ankenbrandt, and J. P. Delahaye, "A muon collider as a higgs factory", in Proc. 4th Int. Particle Accelerator Conf. (IPAC'13), (Shanghai, China), JACoW Publishing, pp. 1472-1474.

[2] A. D. Rujula, M. Gavela, and P. Hernández, "Neutrino oscillation physics with a neutrino factory," Nucl. Phys. B, vol. 547, pp. 21-38, 1999. doi:10.1016/S0550-3213(99)00070-X.

[3] MICE Collaboration, "Demonstration of cooling by the Muon Ionization Cooling Experiment”, Nature, vol. 578, pp. 53-59, 2020. doi:10.1038/s41586-020-1958-9

[4] MuScat Collaboration, "The scattering of muons in low Z materials", Nucl. Instrum. Meth. Sec. A, vol. 83, pp. 492-504, 2005. doi:10.1016/j.nimb.2006.05.006

[5] M. Tanabashi et al., "Review of particle physics", Phys. Rev. D, vol. 98, Sec. 33, 2018. doi:10.1103/PhysRevD.98.030001

[6] S. Agostinelli et al., "Geant4-a simulation toolkit", Nucl. Instrum. Methods Phys. Res. A, vol. 506, no. 3, pp. 250- 303, 2003. doi:10.1016/S0168-9002(03)01368-8.

[7] H. A. Bethe, "Molière's theory of multiple scattering", Phys. Rev. E, vol. 89, pp. 1256-1266, 1953. doi:10.1103/PhysRev.89.1256 